\title{
Multimodal near-infrared-emitting PluS Silica nanoparticles with fluorescent, photoacoustic, and photothermal capabilities
}

This article was published in the following Dove Press journal:

International Journal of Nanomedicine

22 September 2016

Number of times this article has been viewed

\author{
Stefania Biffi' \\ Luca Petrizza ${ }^{2}$ \\ Chiara Garrovo' \\ Enrico Rampazzo 2 \\ Laura Andolfi ${ }^{3}$ \\ Pierangela Giustetto ${ }^{4}$ \\ Ivaylo Nikolov ${ }^{5}$ \\ Gabor Kurdi ${ }^{5}$ \\ Miltcho Boyanov Danailov ${ }^{5}$ \\ Giorgio Zauli' \\ Paola Secchiero ${ }^{6}$ \\ Luca Prodi \\ 'Institute for Maternal and Child \\ Health - IRCCS "Burlo Garofolo", \\ Trieste, ${ }^{2}$ Department of Chemistry \\ "G Ciamician", University of Bologna, \\ Bologna, ${ }^{3}$ IOM-CNR TASC Laboratory, \\ Basovizza, Trieste, ${ }^{4}$ Ephoran - Multi- \\ Imaging Solutions, Bioindustry \\ Park Silvano Fumero, Torino, \\ ${ }^{5}$ Elettra-Sincrotrone Trieste, Trieste, \\ ${ }^{6}$ Department of Morphology, Surgery \\ and Experimental Medicine and \\ LTTA Centre, University of Ferrara, \\ Ferrara, Italy
}

Purpose: The aim of the present study was to develop nanoprobes with theranostic features, including - at the same time - photoacoustic, near-infrared (NIR) optical imaging, and photothermal properties, in a versatile and stable core-shell silica-polyethylene glycol (PEG) nanoparticle architecture.

Materials and methods: We synthesized core-shell silica-PEG nanoparticles by a one-pot direct micelles approach. Fluorescence emission and photoacoustic and photothermal properties were obtained at the same time by appropriate doping with triethoxysilane-derivatized cyanine 5.5 (Cy5.5) and cyanine 7 (Cy7) dyes. The performances of these nanoprobes were measured in vitro, using nanoparticle suspensions in phosphate-buffered saline and blood, dedicated phantoms, and after incubation with MDA-MB-231 cells.

Results: We obtained core-shell silica-PEG nanoparticles endowed with very high colloidal stability in water and in biological environment, with absorption and fluorescence emission in the NIR field. The presence of Cy5.5 and Cy 7 dyes made it possible to reach a more reproducible and higher doping regime, producing fluorescence emission at a single excitation wavelength in two different channels, owing to the energy transfer processes within the nanoparticle. The nanoarchitecture and the presence of both Cy5.5 and Cy 7 dyes provided a favorable agreement between fluorescence emission and quenching, to achieve optical imaging and photoacoustic and photothermal properties.

Conclusion: We obtained rationally designed nanoparticles with outstanding stability in biological environment. At appropriate doping regimes, the presence of Cy5.5 and Cy7 dyes allowed us to tune fluorescence emission in the NIR for optical imaging and to exploit quenching processes for photoacoustic and photothermal capabilities. These nanostructures are promising in vivo theranostic tools for the near future.

Keywords: dye-doped nanoparticles, optical imaging, NIR imaging, photoacoustic imaging, photothermal therapy, PEGylated nanoparticles

\section{Introduction}

Near-infrared (NIR)-emitting nanoparticles (NPs) have been recently investigated as new contrast enhancers for either photoacoustic (PA) or fluorescence in vivo imaging. ${ }^{1-6}$ Photoacoustic imaging (PAI) is based on laser-generated ultrasounds and has emerged over the last decade as a hybrid imaging modality - combining the high-contrast and spectroscopic-based specificity of optical imaging with the high spatial resolution of ultrasound imaging. ${ }^{7}$ On the other hand, because of its high sensitivity, fluorescence optical imaging (FOI) has been increasingly applied to assess tissue pathology, in image-guided surgery, ${ }^{8}$ and for sentinel lymph node fluorescence mapping;,${ }^{9,10}$ several
Correspondence: Luca Prodi Department of Chemistry "G Ciamician", University of Bologna, Via Selmi 2,

40 I 26 Bologna, Italy

Tel +3905 I 209948 I

Fax +3905I 2099456

Email luca.prodi@unibo.it 
intraoperative NIR fluorescence systems are now available for preclinical and clinical studies and some of these systems have been approved by the US Food and Drug Administration (FDA) for use in humans. ${ }^{8}$ Moreover, high-resolution FOI systems for confocal laser endomicroscopy (ie, Mauna Kea fiber-optic system and Pentax/Optiscan confocal endoscope) have been commercialized and have received FDA approval for cancer detection. ${ }^{11}$

The need to combine morphofunctional information in vivo has driven recent efforts for complementing PAI with FOI approaches, particularly by developing suitable dual-modality probes and optical devices for clinical applications. ${ }^{12-14}$ In this regard, multimodal imaging may greatly take advantage of the development of NP-based contrast agents that can combine different physical imaging properties on the same nanoplatform. ${ }^{15}$

Multimodal NIR-emitting NPs for in vivo PAI and FOI primarily need to have a high molar absorption coefficient in the NIR region: PAI and FOI in fact benefit from efficient light absorption in this spectral region. ${ }^{16,17}$ Fluorescence quantum yield has on the other hand an opposite effect on the two imaging modalities. Basically, fluorescence intensity depends on the fluorescence quantum yield $(\Phi)$, whereas the PA signal is typically generated by the remaining energy $(1-\Phi) .{ }^{18}$ However, since dyes emitting in the NIR have $\Phi$ typically $<10 \%$, NIR fluorescent probes can be conveniently used for PAI: a significant improvement of the fluorescent quantum yield (for example, from $5 \%$ to $10 \%$ ) produces only a very limited PA signal cut (the heat released ranging from $95 \%$ to $90 \%$ of the total energy), allowing the possibility to use the same system for both techniques. Additionally, the efficient and rapid conversion of the absorbed photon energy into heat leads to the possible use of the NIR-emitting NPs as photothermal conversion reagents in photothermal therapy (PTT). This therapeutic approach is extremely promising and for this reason is under extensive preclinical and clinical investigations for the treatment of various medical conditions, including cancer. ${ }^{19,20}$

The current understanding of the relationship between optical properties of the NIR-emitting NPs, NP heating efficiency, and their applicative potential in the medical field as theranostic probes is limited. In this context, in the present study, we evaluated both the PA and fluorescence capabilities of dye-doped NIR-pluronic-silica NPs (NIR-PluS NPs), which display a tunable intensity profile across the NIR spectrum. ${ }^{21-23}$ Moreover, using in vitro cell models, the NIR-PluS NPs were investigated as potential PTT material based on their strong optical absorption properties.

\section{Materials and methods}

\section{Chemicals}

The 1-Iodopropane (99\%), malonaldehyde bis(phenylimine) monohydrochloride (97\%), acetic anhydride ( $\geq 99 \%)$, 6-bromohexanoic acid (97\%), dimethylformamide ( $\geq 99.8 \%$, over molecular sieve, $\mathrm{H}_{2} \mathrm{O} \leq 0.01 \%$ ), pyridine ( $\geq 99.8 \%$, over molecular sieve, $\mathrm{H}_{2} \mathrm{O} \leq 0.005 \%$ ), 1 -[bis(dimethylamino) methylene]-1H-1,2,3-triazolo[4,5-b]pyridinium 3-oxide hexafluorophosphate (97\%), citric acid ( $\geq 99.5 \%)$, IR-813 perchlorate (80\%), 3-mercaptopropionic acid ( $\geq 99 \%)$, acetonitrile ( $\mathrm{MeCN}, 99.8 \%$ ), dichloromethane ( $\geq 99.8 \%$ ), Pluronic F127, $N, N, N^{\prime}, N^{\prime}$-tetramethyl-O-(N-succinimidyl) uronium tetrafluoroborate $(97 \%)$, sodium sulfate ( $\geq 99 \%)$, tetraethyl orthosilicate (99.99\%), chlorotrimethylsilane ( $\geq 98 \%)$, acetic acid (HOAc, $\geq 99.7 \%$ ), reagent-grade ethyl acetate (EtOAc), dichloromethane, methanol $(\mathrm{MeOH})$, diethyl ether $\left(\mathrm{Et}_{2} \mathrm{O}\right)$, acetone $\left(\mathrm{Ac}_{2} \mathrm{O}\right)$, and ethanol $(\mathrm{EtOH})$ were purchased from Sigma-Aldrich Co. (St Louis, MO, USA). The 1,1, 2-Trimethylbenz $[e]$ indole ( $\geq 98 \%$ ), triethylamine ( $\geq 99.5 \%$ ), (3-aminopropyl)triethoxysilane ( $\geq 98 \%$ ), N,N-diisopropylethylamine ( $\geq 98 \%$ ), $\mathrm{NaCl}$, and silica on TLC Alu foils ( $4 \times 8 \mathrm{~cm}$, with fluorescent indicator $254 \mathrm{~nm}$ ) were purchased from Sigma-Aldrich Co. (St Louis, MO, USA). UF tubes (Sigma-Aldrich Co.), Amicon Ultra-0.5 mL, cutoff $100 \mathrm{kDa}$, were purchased from EMD Millipore (Billerica, MA, USA). Dialysis was performed versus water at room temperature under gentle stirring with regenerated cellulose dialysis tubing (Sigma-Aldrich Co., molecular weight cutoff $>12 \mathrm{kDa}$, average diameter $33 \mathrm{~mm}$ ).

\section{Synthesis of NIR-PluS NPs}

The synthetic scheme, applied for the preparation of core-shell silica-polyethylene glycol (PEG) dye-doped NPs, is shown in Figure 1. Preparation and morphological characterization were carried out adapting previously reported procedures..$^{21,22}$ For the preparation of NIR-PluS NPs (Table 1), $100 \mathrm{mg}$ of Pluronic F127 and the desired amount of alkoxysilane dye(s) were solubilized with a small amount $(\sim 1.0 \mathrm{~mL})$ of dichloromethane in an $8 \mathrm{~mL}$ glass scintillation vial. The solvent was then evaporated from the homogeneous solution under vacuum at room temperature. $\mathrm{NaCl}(68 \mathrm{mg})$ was added to the solid residue, and the mixture was solubilized at $25^{\circ} \mathrm{C}$ under magnetic stirring with $1,560 \mu \mathrm{L}$ of $1.0 \mathrm{M}$ acetic acid. Tetraethyl orthosilicate $(180 \mu \mathrm{L}, 0.8 \mathrm{mmol})$ was then added to the resulting aqueous homogeneous solution followed by chlorotrimethylsilane $(10 \mu \mathrm{L}, 0.08 \mathrm{mmol})$ after 180 minutes. The mixture was kept under stirring for 48 hours at $25^{\circ} \mathrm{C}$ before dialysis treatments. The dialysis purification steps were 
A

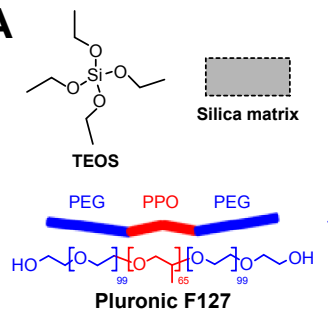

B
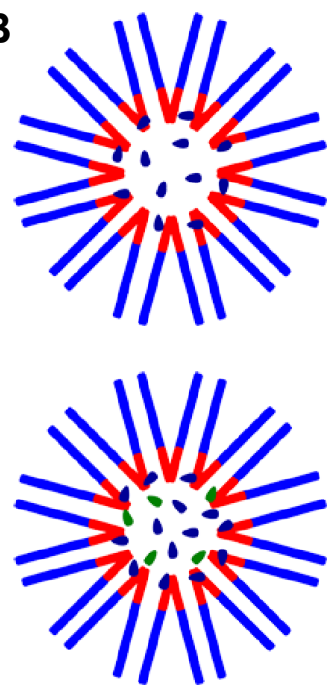

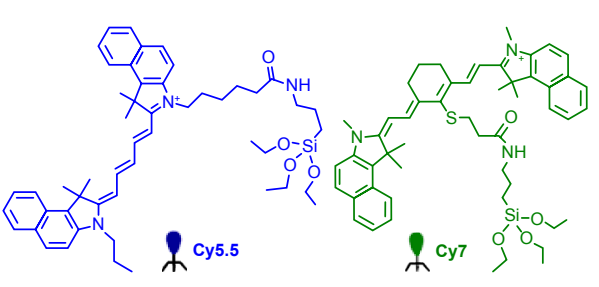

C

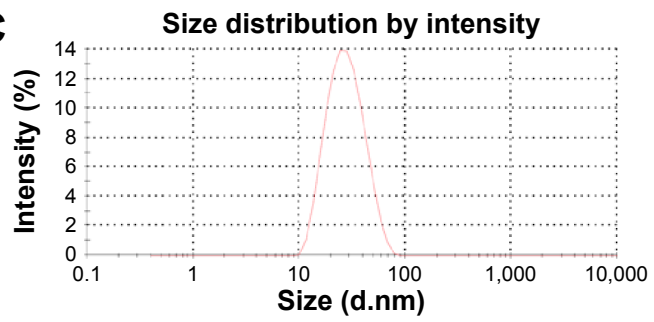

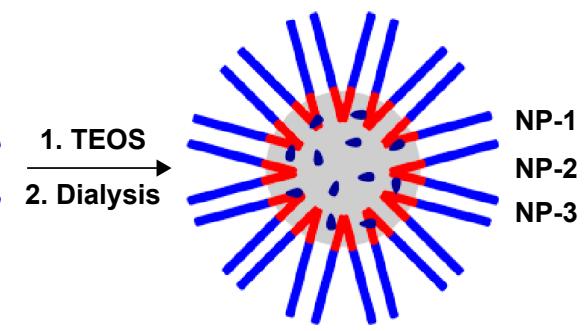

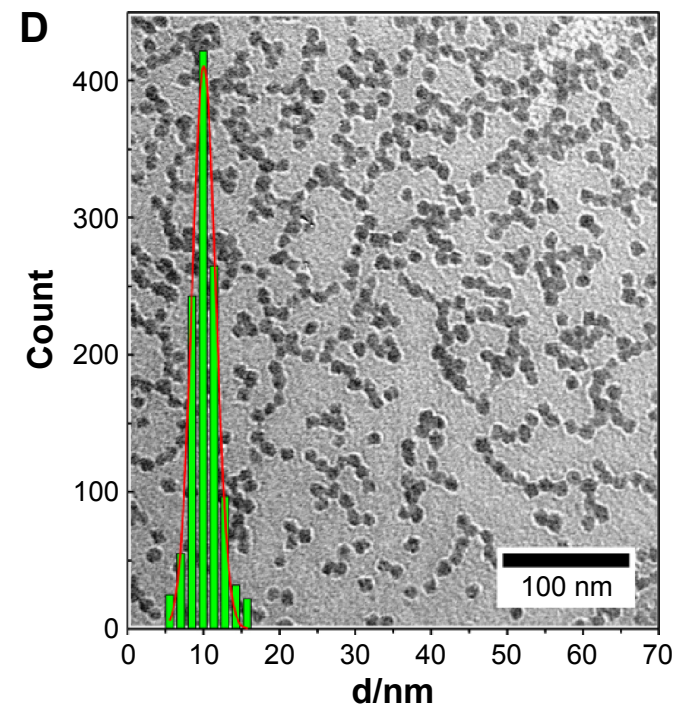

Figure I Synthesis and morphological characterization of NIR-PluS NPs.

Notes: (A) Main chemical components; (B) schematic representation of NIR-PluS NPs synthesis; (C) representative DLS hydrodynamic diameter distribution in water; and (D) representative TEM image with core diameter distribution.

Abbreviations: NIR, near infrared; NIR-PluS NPs, NIR-emitting pluronic-silica nanoparticles; TEM, transmission electron microscope; TEOS, tetraethyl orthosilicate; NP, nanoparticle; DLS, dynamic light scattering.

carried out versus water on a precise amount of NP solution $(1,500 \mu \mathrm{L})$ finally diluted to a total volume of $10.0 \mathrm{~mL}$ with water. The final concentration of the NP solution was measured taking into account the volume after the dialysis.

\section{FOl analysis}

Equimolar aliquots $(10 \mu \mathrm{M})$ of the NIR-PluS NP preparatives were placed on a paper substrate and analyzed using a timedomain fluorescence imager Optix MX2 (ART Advanced
Research Technologies, Montreal, QC, Canada), as previously described. ${ }^{5}$ Briefly, the Optix imaging system data were analyzed using OptiView (ART Advanced Research Technologies) to calculate the fluorescence intensity and decay lifetimes of the NIR-PluS NPs. Optical imaging results were analyzed by reporting fluorescence intensity values in normalized counts representing the photon count for unit excitation laser power and unit exposure time, allowing comparison among different images.

Table I Doping characteristics of the NIR-PluS NPs presented in this study

\begin{tabular}{|c|c|c|c|c|c|c|c|c|}
\hline \multirow[t]{2}{*}{ Sample } & \multicolumn{2}{|l|}{$\%$ dye $^{a}$} & \multicolumn{2}{|l|}{ Cy5.5 } & \multicolumn{2}{|l|}{$\mathrm{Cy} 7^{\mathrm{b}}$} & \multicolumn{2}{|c|}{ Dye/NP } \\
\hline & Cy5.5 & Cy7 & $(\mu \mathrm{mol})$ & $(\mu \mathrm{mol})$ & (mg) & (mg) & Cy5.5 & Cy7 \\
\hline NP-I & 0.5 & - & 4 & - & - & 3.3 & 0.5 & - \\
\hline NP-2 & I & - & 8 & - & - & 6.6 & I & - \\
\hline NP-3 & 5 & - & 40 & - & - & 33 & II & - \\
\hline NP-4 & - & 1 & - & 8 & 6.8 & - & - & 19 \\
\hline NP-5 & 0.5 & I & 4 & 8 & 6.8 & 3.3 & 7 & 4 \\
\hline NP-6 & I & 0.5 & 8 & 4 & 3.4 & 6.6 & 10 & 3 \\
\hline NP-7 & I & I & 8 & 8 & 6.8 & 6.6 & 15 & 6 \\
\hline
\end{tabular}

Notes: a Doping ratio $[\mathrm{mol}(\mathrm{dye}) / \mathrm{mol}(\mathrm{TEOS})] \times 100 .{ }^{\mathrm{b}} \mathrm{Cy} 5.5$ and $\mathrm{Cy} 7$ were introduced in the reaction mixtures using pristine dichloromethane solutions.

Abbreviations: NIR, near infrared; NIR-PluS NPs, NIR-emitting pluronic-silica nanoparticles; NP, nanoparticle; Cy5.5, cyanine 5.5; Cy7, cyanine 7; TEOS, tetraethyl orthosilicate. 


\section{PAl analysis}

The PA signals were captured using a microultrasound array transducer (LZ-250), which operated between $13 \mathrm{mHz}$ and $24 \mathrm{mHz}$ with a resolution of $40 \mu \mathrm{m}$ on the Vevo-2100 (FujiFilm VisualSonics Inc., Toronto, ON, Canada) and a $20 \mathrm{~Hz}$ tunable laser with a wavelength range of 680-970 nm. The central frequency applied was $21 \mathrm{mHz}$, the PA setting parameters had a power output of $100 \%$, B-Mode gain $18 \mathrm{~dB}$, and PA gain $40 \mathrm{~dB}$ with high sensitivity; this setup had been applied to all acquisitions, with a persistence of level 6 .

The phantom for in vitro tests (Supplementary materials) is made of polyurethane tubes (outer diameter $0.9 \mathrm{~mm}$ and inner diameter $0.6 \mathrm{~mm}$ ) submerged in a vinyl and borax gel, water, and liquid starch (the proportional percentage of each component is $25 \%$ ); all components were collected in a PVC cylindrical container. B-mode images were acquired thanks to the acoustic characteristics of the materials used and at the same time in PA modality. Images were acquired with a signal noise ratio value of $22.8 \mathrm{~dB}$ (obtained by randomly selecting five different points on the PA signal and background). The acquisitions were made along the major axis of the tubes, obtaining contents tube axial section images.

Each PA intensity value was calculated as a function of the region of interest outlined on the area of analysis, by identifying the region where the PA signal was present. The PA intensity was the result of the average of five random samples, measured in different points of the sample tube.

\section{Cells}

Epithelial breast cancer cells MDA-MB-231 (purchased from American Type Culture Collection [ATCC], Manassas, VA, USA) were cultured in DMEM (Dulbecco's Modified Eagle's medium)-F12 1:1 supplemented with 10\% FBS (fetal bovine serum), $1 \% \mathrm{~L}$-glutamine, and 1\% penicillin/streptomycin (all from Euroclone, Milano, Italy).

\section{Cell fluorescence imaging}

MDA-MB-231 cells were seeded onto six-well cover glass slides (Thermo Fisher Scientific, Waltham, MA, USA) at a density of $15 \times 10^{4} /$ well. After 24 hours, the medium was replaced by, 1) fresh medium (control) or, 2) fresh medium containing NIR-PluS NPs $100 \mathrm{nM}$. After 24-hour incubation, the cells were washed twice with phosphate-buffered saline (PBS) and fixed with paraformaldehyde $4 \%$ for 30 minutes. Then the cells were washed twice with PBS and mounted on glass slides using a glycerol-based preventing photobleaching mounting media containing DAPI (Sigma-Aldrich Co.). Finally, the cells were observed using a fluorescence microscope: fluorescent images were acquired using an inverted microscope with a CCD camera (DVC-1412AM monochrome digital camera $\mathrm{QE}>62 \%$ at $550 \mathrm{~nm}$ ) and the objectives lens $10 \times$ and $63 \times$ (numerical aperture 1.63) immersion oil.

\section{Photothermal assays}

NIR-PluS NPs were characterized for heating properties using an instrument setup previously reported. ${ }^{24}$ NIR-PluS NP preparations were disposed in a final volume of $100 \mu \mathrm{L}$ in a 96-well sterile plate. The light source used in the experiments was a near-infrared fiber-coupled diode laser LIMO25F200-DL808 (from LIMO Lissotschenko Mikrooptik GmbH, Dortmund, Germany). It was integrated in an IB Photonics LDD80C controller, which provided fine adjustment of the output power and thermal stabilization of the laser. The center wavelength of the output radiation was $808 \mathrm{~nm}$, and the maximum output power was $25 \mathrm{~W}$. In this experiment, it was used with an optical fiber with $400 \mu \mathrm{m}$ core diameter. In the current optical setup, the laser spot diameter at the sample holder surface was $\sim 4.2 \mathrm{~mm}$, measured at $1 / e^{2}$ intensity level. A laser power density of $36 \mathrm{~W} / \mathrm{cm}^{2}$ (corresponding to $5 \mathrm{~W} /$ well) was applied. The heating effect of the laser on the sample was measured using thermal imaging camera Flir InfraCam (Flir System, Inc., Boston, MA, USA) having a temperature measurement range from $-10^{\circ} \mathrm{C}$ to $+350^{\circ} \mathrm{C}$ and sensitivity better than $0.2^{\circ} \mathrm{C}$. The resolution of the sensor was $120 \times 120$ pixels. For experiments in cell models, MDA-MB231 cells, seeded in 96-well flat bottom plates, were exposed to NIR-PluS NPs $100 \mathrm{nM}$ for 24 hours before irradiation for 1 minute per well. After laser exposure, cells were incubated for a further 24 hours and the cellular response to photothermal ablation was measured using an MTT assay.

\section{Statistical analysis}

Results from at least three independent experiments are reported as the mean \pm SD and analyzed for statistical significance by the two-tailed Student's $t$-test and Mann-Whitney rank-sum test. Differences were considered significant when $P$-value was $<0.05$.

\section{Results and discussion}

Fluorescent PluS NPs have recently attracted increasing attention in the biomedical field because of their unique morphological and optical properties. PluS NPs are core-shell silica-PEG NPs synthesized with a one-pot mild condition synthetic approach based on the formation of Pluronic ${ }^{\circledR}$ F 127 micelles in aqueous solution. They are composed of a $\sim 10 \mathrm{~nm}$ silica core surrounded by a $\sim 5-8 \mathrm{~nm}$ PEG shell, a hybrid 
structure conferring very high monodispersion and colloidal stability in aqueous solution and physiological media, with low adhesion properties toward proteins (Supplementary materials). ${ }^{9,25}$ Covalent doping of the silica core with alkoxysilane derivatized dyes produces PluS NPs that are very bright, with luminescent properties that were exploited in several fields, ${ }^{26}$ such as the development of chemosensors, ${ }^{27,28}$ fluorescent photoswitchable NPs, ${ }^{29}$ NPs for electrochemiluminescence applications, ${ }^{30,31}$ in vivo FOI, ${ }^{5,9}$ and targeted fluorescence imaging. ${ }^{9,32}$ Our previous findings have documented efficient cell internalization and lack of cytotoxicity of PluS NPs in some in vitro and in vivo assays. ${ }^{33}$

In this study, we have optimized fluorescent PluS NPs for PA and photothermal applications, by applying protocols of synthesis and of morphological characterization as shown in Figure 1 (Tables S1-S2 and Figures S2-S16).

NIR cyanine 5.5 (Cy5.5) and cyanine 7 (Cy7) dyes (Figures 1 and $\underline{\text { S17-S42) }}$ were simultaneously covalently embedded into the PluS NPs to develop a bright NIR emitting NP material free of dye leaching with both absorption and emission in the NIR range. In particular, by varying the doping ratio of the two dyes (Table 1), absorption and emission profiles could be tuned to have the NIR-PluS NPs exhibiting multiple emission wavelengths by single wavelength excitation (Figures 2A and S43-S49).

The photophysical properties of the different NIR-PluS NPs are listed in Table 2. For all the NIR-PluS NPs, the absorption spectrum is the result of the contribution of the two dyes that, knowing the concentration of the NPs, can be used to estimate the mean number of dyes contained in each NP, ${ }^{21}$ with small differences that can be attributed to the formation of aggregates, as already observed in other cases. ${ }^{34}$ It is worth noting that the molar absorption coefficient observed for the most highly doped systems in the NIR region is $>10^{6}$ $\mathrm{M}^{-1} \mathrm{~cm}^{-1}$, which represents a very remarkable feature, beneficial for all the applications discussed here, namely FOI, PAI,
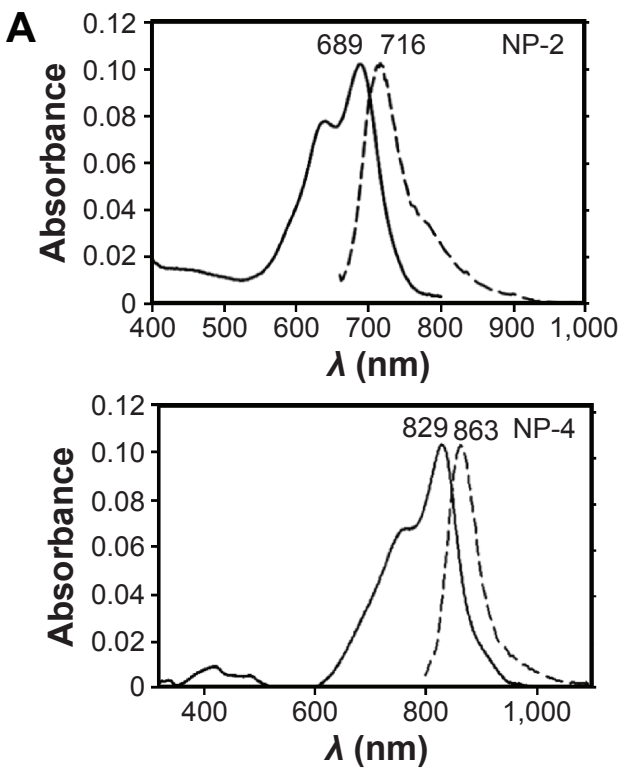

B
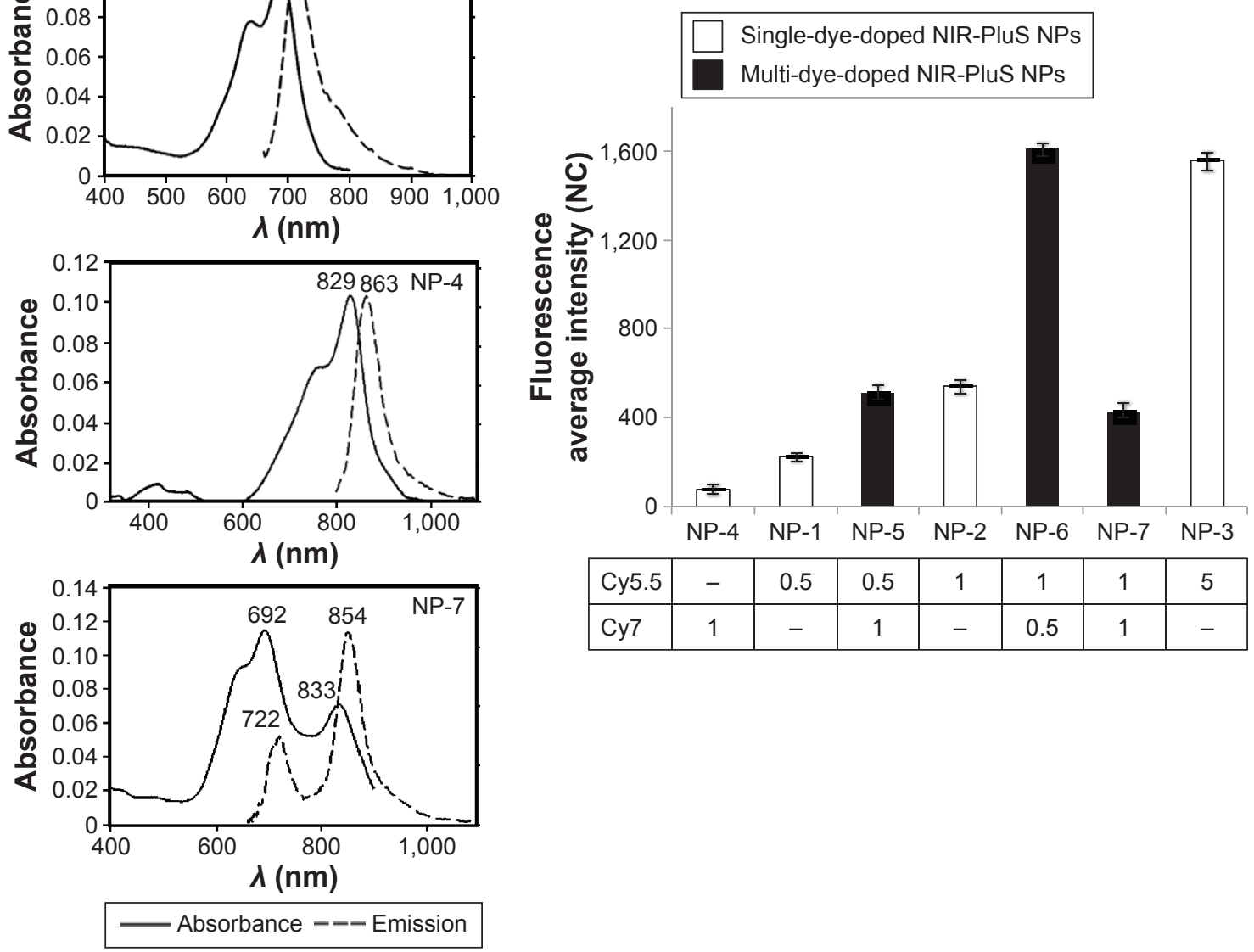

Figure 2 Comparison of fluorescence properties of NIR-PluS NP preparations.

Notes: (A) UV-Vis spectra. (B) Equimolar aliquots of the NIR-PluS NP preparatives were placed on a paper substrate before analysis of the fluorescence with a time-domain fluorescence imager. Data are expressed as mean \pm SD of three independent experiments.

Abbreviations: NIR, near infrared; NIR-PluS NPs, NIR-emitting pluronic-silica nanoparticles; NP, nanoparticle; NC, normalized counts; Cy5.5, cyanine 5.5; Cy7, cyanine 7. 
Table 2 Photophysical properties of the NIR-PluS NPs presented in this study

\begin{tabular}{|c|c|c|c|c|c|c|c|c|c|c|c|}
\hline Sample & $\begin{array}{l}\lambda_{\mathrm{abs}} \max \\
(\mathrm{nm})\end{array}$ & $\begin{array}{l}\varepsilon\left(\lambda_{\max }\right) \\
\left(\mathrm{M}^{-1} \mathbf{c m}^{-1}\right)\end{array}$ & $\begin{array}{l}\lambda_{\mathrm{em}} \max \\
(\mathrm{nm})\end{array}$ & $\Phi_{\mathrm{em}}$ & $\Phi_{\text {em }}{ }^{d}$ & $\Phi_{\mathrm{em}}{ }^{\mathrm{e}}$ & $\Phi_{\text {em }}{ }^{f}$ & $\Phi_{\mathrm{em}}^{\mathrm{g}}$ & $\tau(\mathbf{n s})^{\mathrm{h}}$ & $\tau(\mathbf{n s})^{i}$ & $\eta_{\mathrm{ET}}(\%)$ \\
\hline NP-I & 688 & 64,000 & $712^{\mathrm{a}}$ & 0.18 & - & - & - & - & I. 4 & - & - \\
\hline NP-2 & 689 & 136,000 & $716^{a}$ & 0.18 & - & - & - & - & 1.2 & - & - \\
\hline NP-3 & 691 & $\mathrm{I}, \mathrm{I} 40,000$ & $72 I^{a}$ & 0.32 & - & - & - & - & 0.8 & - & - \\
\hline NP-4 & 829 & $2,582,000$ & $863^{b}$ & 0.06 & - & - & - & - & - & - & - \\
\hline \multirow[t]{2}{*}{ NP-5 } & 692 & 705,000 & 720 & - & 0.06 & 0.02 & 0.04 & 0.11 & 0.5 & 0.5 & 64 \\
\hline & 830 & 603,000 & $856^{c}$ & & & & & & & & \\
\hline \multirow[t]{2}{*}{ NP-6 } & 692 & $1,038,000$ & 719 & - & 0.11 & 0.05 & 0.06 & 0.14 & 0.7 & 0.6 & 42 \\
\hline & 830 & 395,000 & $854^{c}$ & & & & & & & & \\
\hline \multirow[t]{2}{*}{ NP-7 } & 692 & I,53।,000 & 722 & - & 0.03 & 0.01 & 0.02 & 0.03 & 0.4 & 0.5 & 67 \\
\hline & 833 & 765,000 & $854^{c}$ & & & & & & & & \\
\hline
\end{tabular}

Notes: ${ }^{\mathrm{a}} \lambda_{\mathrm{ex}}=650 \mathrm{~nm}$; ${ }^{\mathrm{b}} \lambda_{\mathrm{ex}}=750 \mathrm{~nm}$; ${ }^{\mathrm{C}} \lambda_{\text {ex }}=640 \mathrm{~nm}$; dbased on the area underneath the emission spectra of Cy5.5 and Cy7 following the excitation of Cy5.5; ${ }^{\mathrm{b} b a s e d}$ on the area underneath the emission spectrum of Cy5.5 following the excitation of Cy5.5; fbased on the area underneath the emission spectrum of Cy7 following the excitation of Cy5.5; gbased on the area underneath the emission spectrum of Cy7 following the excitation of $\mathrm{Cy} 7 ;{ }^{\mathrm{h}} \lambda_{\mathrm{ex}}=640 \mathrm{~nm} ; \lambda_{\text {em }}=$ emission maximum of $C y 5.5 ;{ }^{\mathrm{i}} \lambda_{\text {ex }}=640 \mathrm{~nm} ; \lambda_{\mathrm{em}}=\mathrm{emission}$ maximum of Cy7.

Abbreviations: NIR, near infrared; NIR-PluS NPs, NIR-emitting pluronic-silica nanoparticles; NP, nanoparticle; Cy5.5, cyanine 5.5; Cy7, cyanine 7.

and PTT. The analysis of fluorescence spectra revealed an unexpected behavior. Typically, the close proximity of fluorescent dyes, even in silica NPs, can lead to self-quenching processes, leading to a decrease in the fluorescence quantum yield and thus in the overall brightness of the system. For this reason, although some strategies can be adopted to overcome this problem, it is in general detrimental to the overall brightness of the system to increase the doping degree beyond a critical value. In contrast, looking at the NPs doped with only one kind of dye, the highest fluorescence quantum yields were reached at the highest dye content, suggesting that in these cases the aggregation could favor the development of fluorescent nanostructures. In the NIR-PluS NPs containing both dyes, the spectral overlap between the absorption of $\mathrm{Cy} 7$ and the emission of Cy5.5 resulted in an energy transfer process from Cy5.5 to Cy7, with efficiencies $\left(\eta_{\mathrm{ET}}\right)$ ranging from $42 \%$ to $67 \%$ (Table 2). While for other applications, as cytometry, we synthesized NIR-PluS NPs in which the energy transfer process was almost complete (efficiencies $>95 \%$ ), ${ }^{35}$ in this case we preferred a design yielding an intermediate behavior to maintain two different fluorescent signals (at $720 \mathrm{~nm}$ and $860 \mathrm{~nm}$, for Cy5.5 and Cy7, respectively), but obtaining an enhancement of the $\mathrm{Cy} 7$ emission intensity even upon excitation at $650 \mathrm{~nm}$ (Cy5.5 excitation) when compared with the single-doped NIR-PluS NPs excited at $750 \mathrm{~nm}$ (Cy7 excitation), as can be observed in Figure 2. Moreover, in these conditions the multiple-doped NIR-PluS NPs developed displayed a narrow-band emission with a large Stokes shift of $\cong 200 \mathrm{~nm}$, which effectively increases the specificity over background emission, avoiding crosstalk between the excitation light and the emitting signals. Although for these NPs it is difficult to discern the effect of aggregation upon changing the amount of dyes, it is important to underline that in particular for NP-5 and NP-6 the high absorption is accompanied by a fluorescence quantum yield that is quite high, at least if compared with the dyes emitting in this spectral region.

After the general photophysical characterization of our NPs, we performed fluorescence imaging using the MDAMB-231 epithelial tumor cells that we conceived as model systems with the aim to preliminarily assess (in vitro) potential applications in PTT, in response to a $680 \mathrm{~nm}$ excitation source. First of all, cell cultures were incubated with the NIR-PluS NPs (NP-7 $100 \mathrm{nM}$ ), and microscopy analyses demonstrated that cells were effective in NIR-PluS NP uptake and could be easily visualized with imaging modality (Figure 3 ).

These results clearly support the potential of using NIR-PluS NPs as bright fluorescent probes in cellular imaging. Of note, besides the enhanced and tunable fluorescence emission properties useful for optical imaging, NIR-PluS NPs can convert the absorbed light into heat via a series of nonradiative processes. The photophysical properties discussed so far are thus also suitable to produce a large PA signal. In particular, as shown in Figure 4A, the PA spectral profiles of NIR-PluS NP preparations indicated their excellent PA signal intensities in PBS. As expected, the PA spectra of NP-5, NP-6, and NP-7 showed two very intense peaks at $680 \mathrm{~nm}$ and $820 \mathrm{~nm}$, corresponding to the absorption of Cy5.5 and Cy7, respectively. The PA signals were also measured in blood to account for the effect of red blood cells absorption (Figure 4B).

The phantom measurements (Figure S1) indicated that laser wavelengths could be selected to provide minimal overlapping between spectra bands of blood and NIRPluS NPs and to achieve the detection of NIR-PluS NPs 

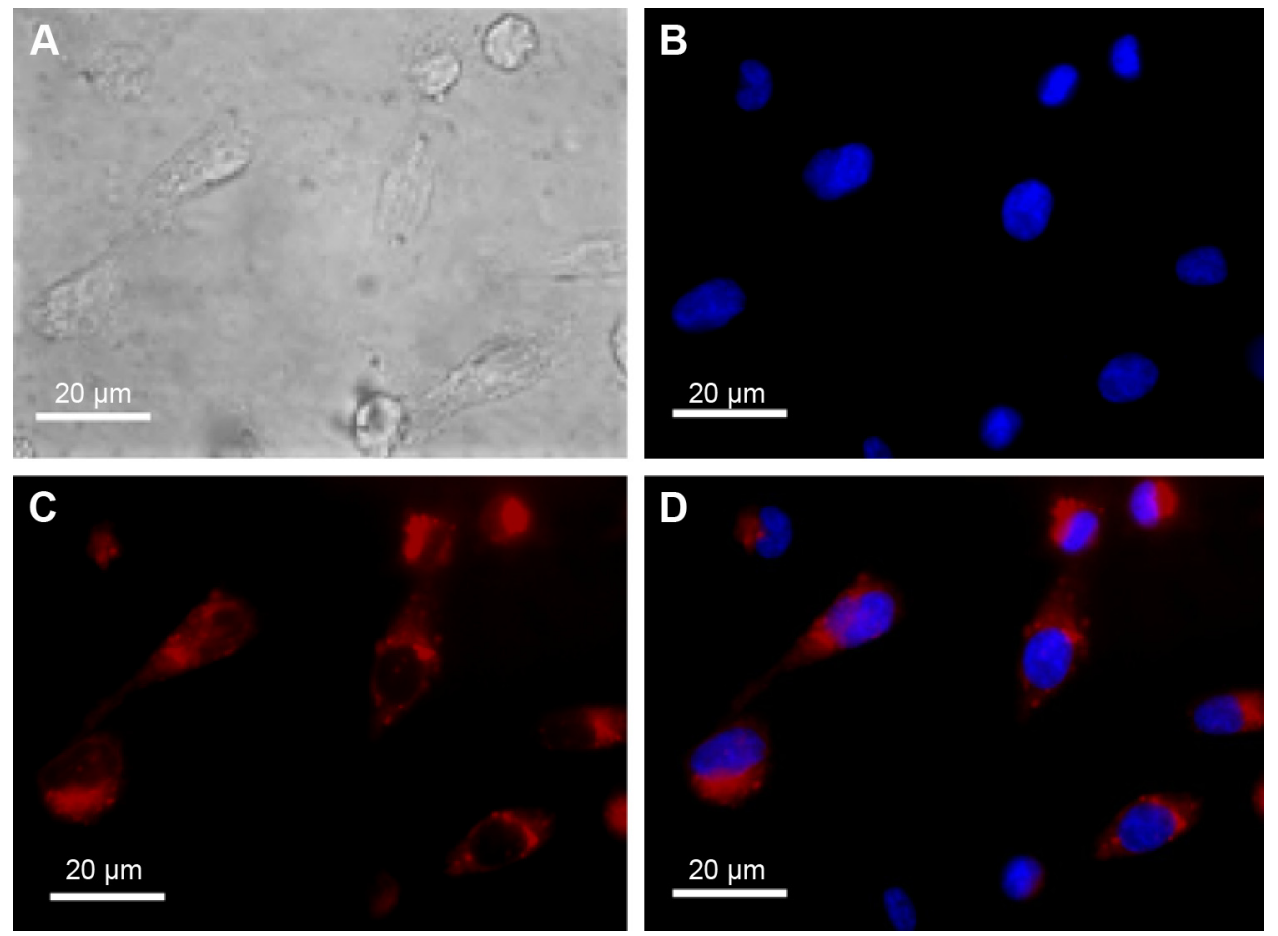

Figure 3 Fluorescence cell imaging.

Notes: MDA-MB-23I cells were incubated with NIR-PluS NPs (NP-7; $100 \mathrm{nM}$ ) at $37^{\circ} \mathrm{C}$ for 24 hours, to allow NIR-PluS NP cell internalization before microscopy analysis. Representative cell images are shown. DAPI staining was carried out to visualize cell nuclei, which appear in blue, while NIR-PluS NPs appear in red. (A) Bright field image;

(B) DAPI nuclear staining; (C) fluorescence emission; and (D) overlaid images (bar $=20 \mu \mathrm{m})$.

Abbreviations: NIR, near infrared; NIR-PluS NPs, NIR-emitting pluronic-silica nanoparticles.

avoiding blood interference (background). Overall, taking advantage of the strong yet spectrally distinct absorption of Cy5.5 and Cy7 in the NIR-infrared range, PA analysis enabled detecting and distinguishing of the contribution of each dye to the signal profile, opening the possibility for multiplexing imaging (Figure 4C). Moreover, the synthetic approach allowed for tuning the PA properties without affecting particle dimension and hydrodynamic properties. This characteristic of NIR-PluS NPs may be beneficial to in vivo multiplexed imaging, as NIR-PluS NPs can be formulated to have distinct PA profiles but the same pharmacokinetic behaviors.
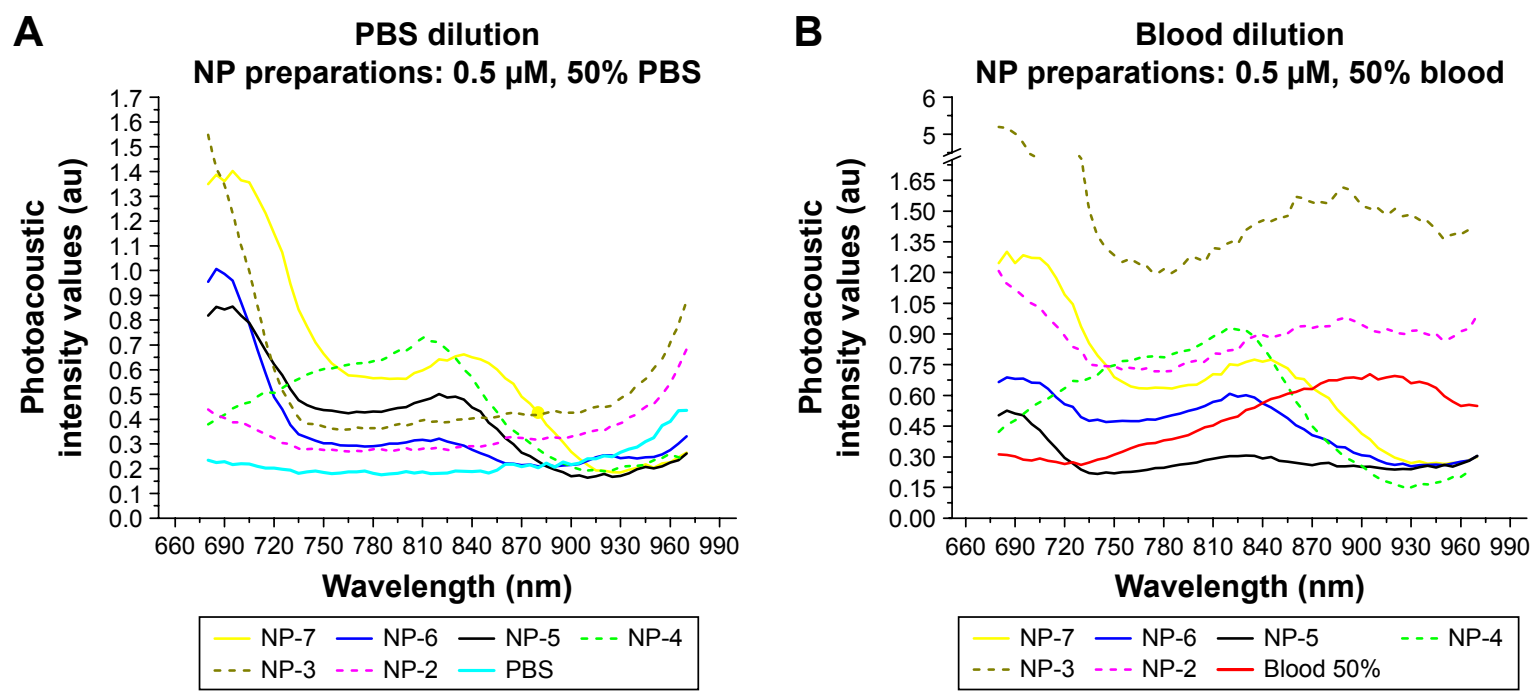

Figure 4 (Continued) 


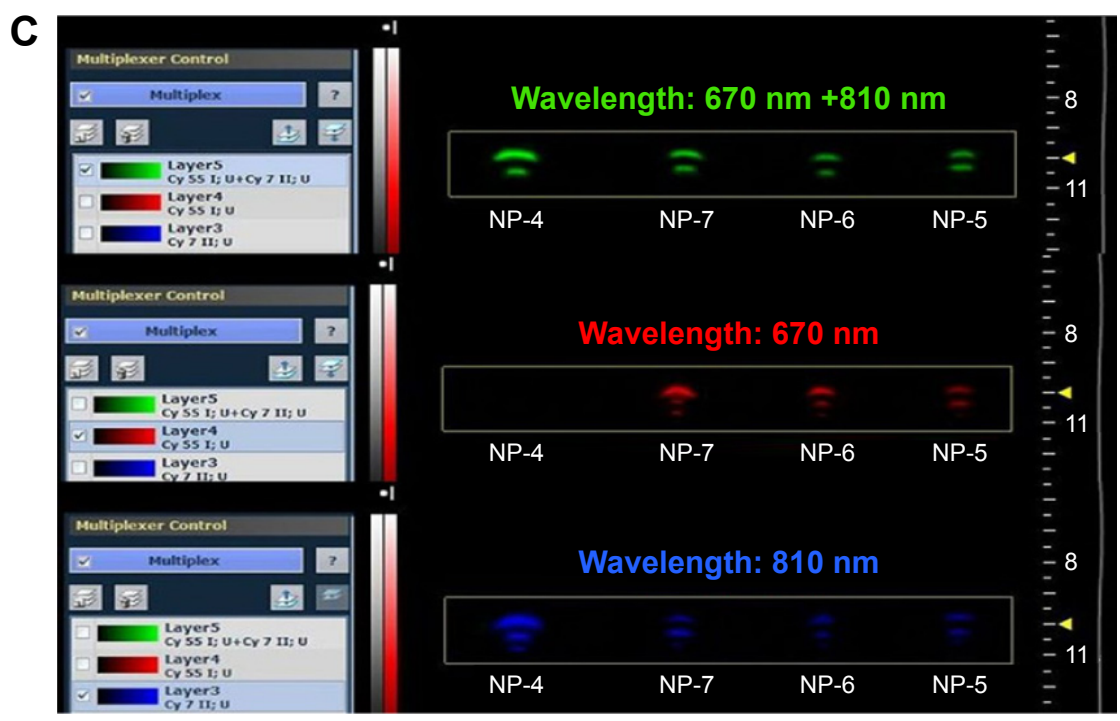

Figure 4 Comparison of PA properties of NIR-PluS NP preparations.

Notes: Representative PA spectra of $0.5 \mu$ M NIR-PluS NP suspensions in (A) PBS and (B) blood. Spectra were measured over the wavelength range of 680-970 nm. (C) Imaging comparison of PA amplitudes of $0.5 \mu$ M NIR-PluS NP suspensions in blood (NP-4, NP-7, NP-6, and NP-5). Multispectral unmixing method was applied to the PA data to visualize the contribution of each dye.

Abbreviations: PA, photoacoustic; NIR, near infrared; NIR-PluS NPs, NIR-emitting pluronic-silica nanoparticles; PBS, phosphate-buffered saline; NP, nanoparticle.

Light-generated heat can also be advantageously used in therapy, and NIR light has been conventionally used for the in vivo PTT treatment of tumors under skin and within tissues, because of its deep penetration and reduced absorption through the high scattering tissue media. In this context, we have evaluated the photothermal capabilities of NIR-PluS NPs, looking at the temperature increase of their aqueous suspensions at different concentrations upon 1-minute irradiation with an $808 \mathrm{~nm}$ continuous wave diode laser with an intensity of $36 \mathrm{~W} / \mathrm{cm}^{2}$ (Figure $5 \mathrm{~A}$ ). In turn, Figure $5 \mathrm{~B}$ depicts the temperature increase of NP-4 and NP-7 suspensions at the lowest concentrations $(0.1 \mu \mathrm{M})$ as a function of irradiation time. In particular, the presence of the Cy7-doping dye granted photothermal properties to the NIR-PluS NP. After 1 minute

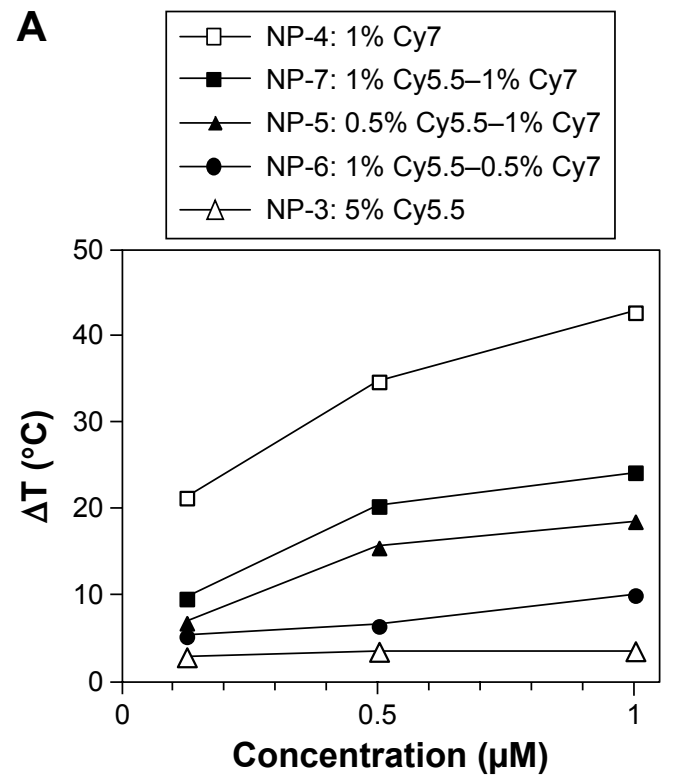

$\mathbf{B}$
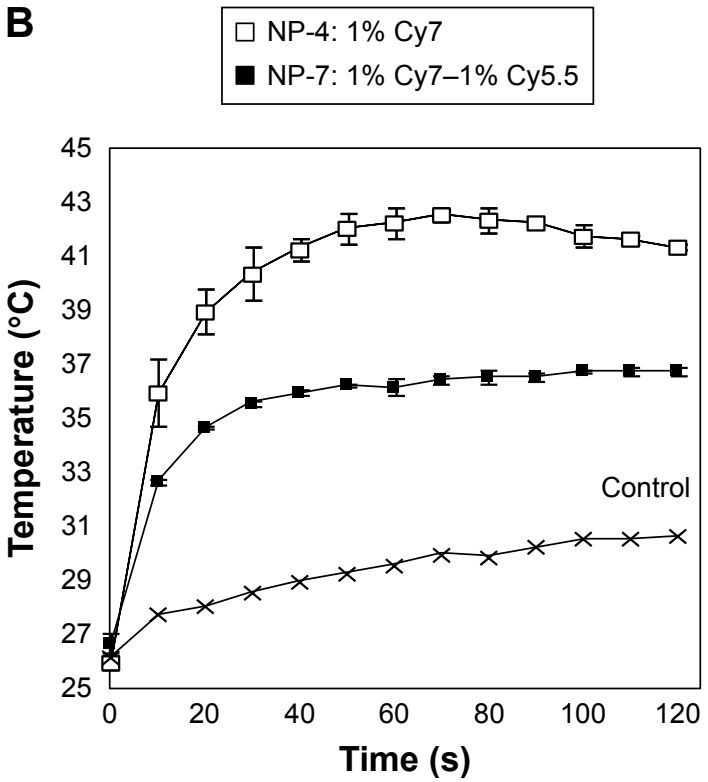

Figure 5 Photothermal properties of NIR-PluS NPs.

Notes: (A) Temperature change ( $\Delta$ temperature) of aqueous suspension of NIR-PluS NP preparations at different concentrations upon irradiation with 808 nm laser $\left(36 \mathrm{~W} / \mathrm{cm}^{2}\right)$ for I minute. (B) Temperature change of aqueous suspensions of NIR-PluS NPs (NP-4 and NP-7, $0.1 \mu$ M), in comparison to the control solution, upon irradiation with $808 \mathrm{~nm}$ laser $\left(36 \mathrm{~W} / \mathrm{cm}^{2}\right)$. The local temperature was measured by an infrared thermal camera and plotted as a function of the irradiation time.

Abbreviations: NIR, near infrared; NIR-PluS NPs, NIR-emitting pluronic-silica nanoparticles; NP, nanoparticle; Cy5.5, cyanine 5.5; Cy7, cyanine 7; s, seconds. 
of irradiation time, a temperature change of $35.5^{\circ} \mathrm{C}$ was measured with NP-4 (1 $\mu \mathrm{M})$ characterized by a 1\% Cy7 doping. In contrast, because of the absence of absorption at the excitation wavelength, no significant temperature change was observed when sample NP-3 with 5\% Cy5.5 doping was irradiated in the same conditions. Consistently, multiple-doped NIR Plus NPs, NP-5, NP-6, and NP-7, showed an intermediate behavior according to the absorption properties at $808 \mathrm{~nm}$.

As a result, NP-4 sample had a heating profile matching more efficiently our experimental setup, with an improved thermal efficiency with respect to the other samples. For these reasons, we have chosen the sample NP-4 for the preliminary evaluation in vitro of the PTT performance with our equipment. For this purpose, MDA-MB-231 cells were incubated with NIR-PluS NPs (NP-4; $100 \mathrm{nM}$ ) for 24 hours and then exposed to an $808 \mathrm{~nm}$ continuous wave diode laser, operating at $36 \mathrm{~W} / \mathrm{cm}^{2}$, for 1 minute. After an additional 24 hours of culture, the cell viability difference between control cells and cells incubated with NIR-Plus NPs was $\sim 43 \%(P<0.01)$ after light treatment (Figure 6). In contrast, we observe only a modest decrease in cell viability $(\sim 10 \%, P<0.01)$ after 48 hours of exposure at the dose of $100 \mathrm{nM}$ of NPs but without light treatment. In cells without NP incubation no effect of irradiation was observed (viability $=99 \%$ of nonirradiated controls). Therefore, these results, while they confirm the lack of cell toxicity of the silica NPs, ${ }^{33}$ clearly show that cell death was induced by the photothermal effect of NIR-PluS NPs.

Similar results were obtained on other relevant cell models (data not shown). Therefore, with localized NIR-PluS NP accumulation, the laser irradiation induced a level of heating, which was sufficient to induce cell death.

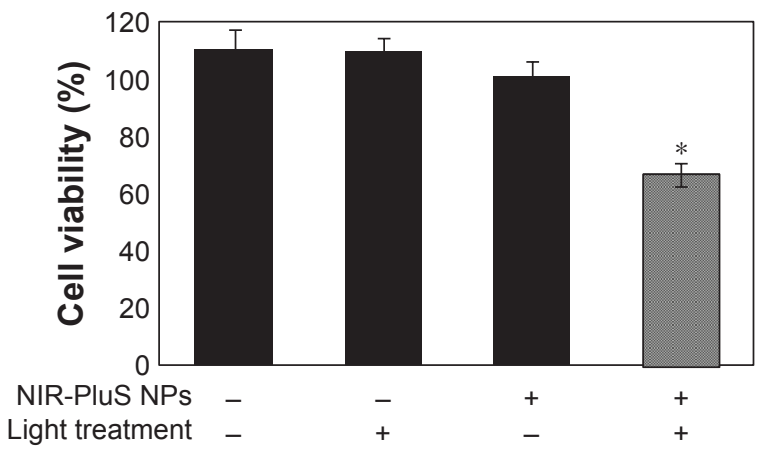

Figure 6 Photothermal ablation of MDA-MB-23। cells in vitro using NIR-PluS NPs. Notes: Cells were incubated with NIR-PluS NPs (NP-4, $100 \mathrm{nM}$ ) at $37^{\circ} \mathrm{C}$ for 24 hours, to allow NIR-PluS NP cell internalization before photothermal heating treatment (irradiation time: I minute, $36 \mathrm{~W} / \mathrm{cm}^{2}$ at $808 \mathrm{~nm}$ ). After an additional 24 hours of culture cell viability was assessed in all the different culture conditions, as indicated. Data are expressed as mean \pm SD of three independent experiments. $* P<0.05$ with respect to control cells.

Abbreviations: NIR, near infrared; NIR-PluS NPs, NIR-emitting pluronic-silica nanoparticles; NP, nanoparticle.

\section{Conclusion}

Our study reports a versatile strategy based on the formation of Pluronic ${ }^{\circledR}$ F127 micelles in aqueous solution, plus the Cy5.5 and Cy7 NIR dyes, that can be covalently embedded within the PluS NPs, to develop NIR emitting multifunctional nanostructured materials. In particular, thanks to their noticeable photophysical features, these NPs showed an excellent brightness in the NIR wavelength range and remarkable PA and photothermal properties. On this basis, we provide preliminary evidence suggesting the potential use of NIR-PluS NPs as bright and nontoxic fluorescent probes in imaging and therapy settings. The concomitant incorporation of Cy5.5 and Cy7 dyes, absorbing in distinct optical channels - centered at $670 \mathrm{~nm}$ and $810 \mathrm{~nm}$ - for both imaging and photothermal treatments, represents an additional feature of great interest for theranostic applications.

\section{Acknowledgment}

This work was supported by: MIUR (PON 01_01078 granted to Luca Prodi) and University of Bologna (FARB project A.10.N2.RICER.FARB2RODAA granted to Luca Prodi).

\section{Author contributions}

Stefania Biffi: designed methods and experiments, analyzed the data, interpreted the biological results, and wrote the paper; Luca Petrizza: performed the synthesis and characterization of nanoparticles and dyes, wrote the supplementary information, interpreted photophysical data, and revised the paper; Enrico Rampazzo: designed the synthesis and characterization of nanoparticles and dyes, interpreted photophysical data, and wrote the paper and supplementary information; Chiara Garrovo: performed in vitro cell experiments, interpreted the biological results, and revised the paper; Laura Andolfi: performed the microcopy analyses, interpreted the biological results, and revised the paper; Pierangela Giustetto: performed PAI experiments, interpreted imaging results, and revised the paper; Ivaylo Nikolov, Gabor Kurdi, and Miltcho Boyanov Danailov: designed the laser irradiation setup, performed the laser irradiation and thermal camera analysis, analyzed photothermal results, and revised the paper; Giorgio Zauli and Paola Secchiero: interpreted the results and reviewed the paper; Luca Prodi: defined the research theme, designed methods and experiments, interpreted the results, and wrote the paper. All authors contributed toward data analysis, drafting and critically revising the paper and agree to be accountable for all aspects of the work.

\section{Disclosure}

The authors report no conflicts of interest in this work. 


\section{References}

1. Wang C, Bao C, Liang S, et al. RGD-conjugated silica-coated gold nanorods on the surface of carbon nanotubes for targeted photoacoustic imaging of gastric cancer. Nanoscale Res Lett. 2014;9(1):264.

2. Jokerst JV, Van de Sompel D, Bohndiek SE, Gambhir SS. Cellulose nanoparticles are a biodegradable photoacoustic contrast agent for use in living mice. Photoacoustics. 2014;2(3):119-127.

3. Beziere N, Lozano N, Nunes A, et al. Dynamic imaging of PEGylated indocyanine green (ICG) liposomes within the tumor microenvironment using multi-spectral optoacoustic tomography (MSOT). Biomaterials. 2015;37:415-424.

4. Li K, Liu B. Polymer-encapsulated organic nanoparticles for fluorescence and photoacoustic imaging. Chem Soc Rev. 2014;43(18):6570-6597.

5. Biffi S, Petrizza L, Rampazzo E, et al. Multiple dye-doped NIR-emitting silica nanoparticles for both flow cytometry and in vivo imaging. RSC Adv. 2014;4(35):18278-18285.

6. Sayag D, Cabon Q, Texier I, et al. Phase-0/phase-I study of dye-loaded lipid nanoparticles for near-infrared fluorescence imaging in healthy dogs. Eur J Pharm Biopharm. 2016;100:85-93.

7. Beard P. Biomedical photoacoustic imaging. Interface Focus. 2011;1(4): 602-631.

8. Mondal SB, Gao S, Zhu N, Liang R, Gruev V, Achilefu S. Real-time fluorescence image-guided oncologic surgery. Adv Cancer Res. 2014;124: $171-211$.

9. Helle M, Rampazzo E, Monchanin M, et al. Surface chemistry architecture of silica nanoparticles determine the efficiency of in vivo fluorescence lymph node mapping. ACS Nano. 2013;7(10):8645-8657.

10. Noh YW, Kong SH, Choi DY, et al. Near-infrared emitting polymer nanogels for efficient sentinel lymph node mapping. ACS Nano. 2012; 6(9):7820-7831.

11. Gabbani T, Manetti N, Bonanomi AG, Annese AL, Annese V. New endoscopic imaging techniques in surveillance of inflammatory bowel disease. World J Gastrointest Endosc. 2015;7(3):230-236.

12. Chen SL, Xie Z, Guo LJ, Wang X. A fiber-optic system for dual-modality photoacoustic microscopy and confocal fluorescence microscopy using miniature components. Photoacoustics. 2013;1(2):30-35.

13. Xi L, Zhou G, Gao N, et al. Photoacoustic and fluorescence imageguided surgery using a multifunctional targeted nanoprobe. Ann Surg Oncol. 2014;21(5):1602-1609.

14. Xi L, Satpathy M, Zhao Q, Qian W, Yang L, Jiang H. HER-2/neu targeted delivery of a nanoprobe enables dual photoacoustic and fluorescence tomography of ovarian cancer. Nanomedicine. 2014;10(3): 669-677.

15. Biffi S, Voltan R, Rampazzo E, Prodi L, Zauli G, Secchiero P. Applications of nanoparticles in cancer medicine and beyond: optical and multimodal in vivo imaging, tissue targeting and drug delivery. Expert Opin Drug Del. 2015;12(12):1837-1849.

16. Oraevsky AA. Contrast agents for optoacoustic imaging: design and biomedical applications. Photoacoustics. 2015;3(1):1-2.

17. Sreejith S, Joseph J, Lin M, et al. Near-infrared squaraine dye encapsulated micelles for in vivo fluorescence and photoacoustic bimodal imaging. ACS Nano. 2015;9(6):5695-5704.

18. Tomasini EP, San Román E, Braslavsky SE. Validation of fluorescence quantum yields for light-scattering powdered samples by laser-induced optoacoustic spectroscopy. Langmuir. 2009;25(10):5861-5868.
19. Kang S, Bhang SH, Hwang S, et al. Mesenchymal stem cells aggregate and deliver gold nanoparticles to tumors for photothermal therapy. ACS Nano. 2015;9(10):9678-9690.

20. Kim HJ, Lee SM, Park KH, Mun CH, Park YB, Yoo KH. Drug-loaded gold/iron/gold plasmonic nanoparticles for magnetic targeted chemophotothermal treatment of rheumatoid arthritis. Biomaterials. 2015; 61:95-102.

21. Rampazzo E, Bonacchi S, Juris R, et al. Energy transfer from silica core-surfactant shell nanoparticles to hosted molecular fluorophores. J Phys Chem B. 2010;114(45):14605-14613.

22. Genovese D, Bonacchi S, Juris R, et al. Prevention of self-quenching in fluorescent silica nanoparticles by efficient energy transfer. Angew Chem Int Ed Engl. 2013;52(23):5965-5968.

23. Genovese D, Rampazzo E, Bonacchi S, Montalti M, Zaccheroni N, Prodi L. Energy transfer processes in dye-doped nanostructures yield cooperative and versatile fluorescent probes. Nanoscale. 2014;6(6): 3022-3036.

24. Kostevsek N, Locatelli E, Garrovo C, et al. The one-step synthesis and surface functionalization of dumbbell-like gold-iron oxide nanoparticles: a chitosan-based nanotheranostic system. Chem Commun (Camb). 2015;52:378-381.

25. Rampazzo E, Boschi F, Bonacchi S, et al. Multicolor core/shell silica nanoparticles for in vivo and ex vivo imaging. Nanoscale. 2012;4(3): 824-830.

26. Bonacchi S, Genovese D, Juris R, et al. Luminescent silica nanoparticles: extending the Frontiers of brightness. Angew Chem Int Ed Engl. 2011;50(18):4056-4066.

27. Rampazzo E, Bonacchi S, Genovese D, et al. A versatile strategy for signal amplification based on core/shell silica nanoparticles. Chemistery. 2011; 17(48):13429-13432.

28. Masseroni D, Biavardi E, Genovese D, Rampazzo E, Prodi L, Dalcanale E. A fluorescent probe for ecstasy. Chem Commun (Camb). 2015;51(64):12799-12802.

29. Genovese D, Montalti M, Prodi L, et al. Reversible photoswitching of dyedoped core-shell nanoparticles. Chem Commun (Camb). 2011;47(39): 10975-10977.

30. Valenti G, Rampazzo E, Bonacchi S, et al. A versatile strategy for tuning the color of electrochemiluminescence using silica nanoparticles. Chem Commun (Camb). 2012;48(35):4187-4189.

31. Zanarini S, Rampazzo E, Bonacchi S, et al. Iridium doped silica-PEG nanoparticles: enabling electrochemiluminescence of neutral complexes in aqueous media. J Am Chem Soc. 2009;131(40):14208-14209.

32. Soster M, Juris R, Bonacchi S, et al. Targeted dual-color silica nanoparticles provide univocal identification of micrometastases in preclinical models of colorectal cancer. Int J Nanomedicine. 2012;7:4797-4807.

33. Rampazzo E, Voltan R, Petrizza L, et al. Proper design of silica nanoparticles combines high brightness, lack of cytotoxicity and efficient cell endocytosis. Nanoscale. 2013;5(17):7897-7905.

34. Rampazzo E, Bonacchi S, Montalti M, Prodi L, Zaccheroni N. Selforganizing core-shell nanostructures: spontaneous accumulation of dye in the core of doped silica nanoparticles. J Am Chem Soc. 2007; 129(46):14251-14256.

35. Rampazzo E, Bonacchi S, Genovese D, et al. Pluronic-silica (PluS) nanoparticles doped with multiple dyes featuring complete energy transfer. J Phys Chem C. 2014;118(17):9261-9267.
International Journal of Nanomedicine

\section{Publish your work in this journal}

The International Journal of Nanomedicine is an international, peerreviewed journal focusing on the application of nanotechnology in diagnostics, therapeutics, and drug delivery systems throughout the biomedical field. This journal is indexed on PubMed Central, MedLine, CAS, SciSearch $\AA$, Current Contents $\AA /$ Clinical Medicine,

\section{Dovepress}

Journal Citation Reports/Science Edition, EMBase, Scopus and the Elsevier Bibliographic databases. The manuscript management system is completely online and includes a very quick and fair peer-review system, which is all easy to use. Visit http://www.dovepress.com/ testimonials.php to read real quotes from published authors. 